Images of

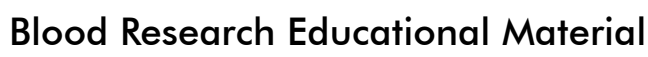

(emo

Volume $54 \cdot$ Number $3 \cdot$ September 2019

https://doi.org/10.5045/br.2019.54.3.164

\title{
Neutrophil-erythrocyte rosettes in direct antiglobulin test-negative autoimmune hemolytic anemia
}

\author{
Hee Sue Park ${ }^{1}$, Kyeong Seob Shin ${ }^{1,2}$, Bo Ra Son ${ }^{1,2}$ \\ ${ }^{1}$ Department of Laboratory Medicine, Chungbuk National University Hospital, ${ }^{2}$ Department of Laboratory Medicine, \\ Chungbuk National University College of Medicine, Cheongju, Korea
}

Received on May 16, 2019; Revised on August 6, 2019; Accepted on August 9, 2019

Correspondence to Bo Ra Son, M.D., Department of Laboratory Medicine, Chungbuk National University College of Medicine, 1 Chungdaero, Seowon-gu, Cheongju-si, Chungbuk 28644, Korea, E-mail: brson@cbungbuk.ac.kr
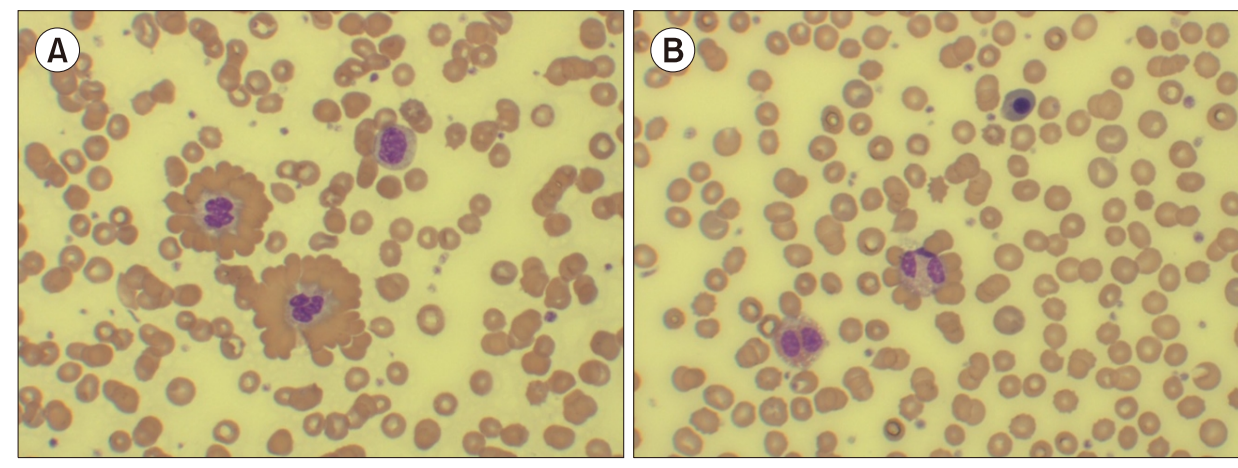

An 83-year-old woman was transferred for antifungal therapy for candidemia that occurred during an intracerebral hemorrhage treatment at another hospital. At admission, complete blood count results were as follows: hemoglobin, $8.8 \mathrm{~g} / \mathrm{dL}$; white blood cell (WBC), $18.51 \times 10^{9} / \mathrm{L}$; neutrophil, $16.66 \times 10^{9} / \mathrm{L}$; platelet, $52 \times 10^{9} / \mathrm{L}$; and elevated C-reactive protein $(\mathrm{CRP})$ and procalcitonin, $32.47 \mathrm{mg} / \mathrm{dL}$ and $6.60 \mathrm{ng} / \mathrm{mL}$, respectively. After anidulafungin treatment, WBC count and CRP level were normalized, but hemoglobin level continued to decrease. Test results for anemia were as follows: ferritin, $741.82 \mathrm{ng} / \mathrm{mL}$; iron, $198 \mu \mathrm{g} / \mathrm{dL}$; transferrin saturation, 87.6\%; total iron-binding capacity, 226 $\mu \mathrm{g} / \mathrm{dL}$; folate, $6.4 \mathrm{ng} / \mathrm{mL}$; vitamin $\mathrm{B} 12,>2,000 \mathrm{pg} / \mathrm{mL}$; lactate dehydrogenase, $538 \mathrm{U} / \mathrm{L}$; total bilirubin, $4.99 \mathrm{mg} / \mathrm{dL}$; collected reticulocyte count, 3.4\%; and haptoglobin, $24 \mathrm{mg} / \mathrm{dL}$. The direct antiglobulin test for IgG+ C3d was negative; hematuria was not observed. Considering these findings, autoimmune hemolytic anemia (AIHA) was suspected, and peripheral blood $(\mathrm{PB})$ smear conclusively showed frequent neutrophil-erythrocyte rosettes [composite image of $\mathrm{PB}$ smear, Wright-Giemsa staining, $\times 400$; (A) red blood cell (RBC) rosettes around neutrophils. (B) Few nucleated RBC were observed, but schistocyte or spherocytes were not observed]. The neutrophil-erythrocyte rosettes formation is a very rare AIHA phenomenon. This phenomenon is caused by the interaction between neutrophil Fc receptors and IgG-decorated RBCs. 